mainly invited reviews, mini-reviews, commentaries and issues of the day. The topics published so far have been very wide ranging, from the application of crystallography in the design of viral agents to the pathogenicity of Entamoeba histolytica and cytokines in helminth infections. Although the authors are all acknowledged experts, their contributions vary considerably in clarity and depth. The obvious comparison must be with Immunology Today, which is much better value for money, with 516 pages in 1992 (compared with 342) and more than twice as many articles. On the other hand, Infectious Agents and Disease is concerned with the real world of immunity to infection and is an attractive and authoritative publication that will appeal to many immunologists, microbiologists, parasitologists and clinicians. Of the three journals, Infectious Agents and Disease is the one most likely to succeed.

F. E. G. Cox is in the Immunology and Infection Group, Division of Life Sciences, King's College London, Campden Hill Road, London W8 $7 A H$, UK.

\section{Muscling in}

\section{Malcolm J. Jackson}

Basic and Applied Myology. Editor-inchief U. Carraro. Unipress, Padova, Italy. 4/yr. Italy IL150,000, elsewhere $\$ 150$ (institutional); Italy IL100,000, elsewhere $\$ 100$ (personal).

Neuromuscular Disorders. Editor-inchief V. Dubowitz. Pergamon. 6/yr. £217, \$335 (institutional); £80, \$123 (personal).

THE first leading article in Basic and Applied Myology (BAM) asks whether the new professional activity of 'myology' is emerging. From the clinical point of view, skeletal muscle has usually been placed in the field of neurology with papers mainly being published in neurological journals. The number of specialist journals for muscle researchers has been small. These two new journals are both seeking to attract papers from the group whose prime area of interest is muscle. Both are interdisciplinary: Neuromuscular Disorders covers both clinical and basic sciences of relevance to the neuromuscular diseases of childhood and adult life, whereas $B A M$ aims to cover basic skeletal-muscle research and its clinical applications.

Muscle contraction has kept traditional physiologists busy for many years, but the past decade has seen many other disciplines looking at this tissue: molecular biologists have played an important role in the elucidation of the underlying defects in Duchenne, Becker and myotonic muscular dystrophies, whereas cell biologists have become fascinated by the techniques and prospects of cell therapy for muscle disorders; even electronic engineers have been involved, developing implantable stimulators for electrical stimulation and transforming skeletal muscle fibre types for cardiac repair.

Both journals attempt to provide a forum for the publication of papers from many different disciplines. Overall there have been more papers on basic science in $B A M$ and, among the original articles and reviews, a high proportion have dealt with the use of skeletal muscle in the assistance of failing cardiac tissue. $B A M$ is well presented, with good-quality electron micrographs and histology plates.

Neuromuscular Disorders is also well presented. It is more clinically oriented, and publishes a wide range of papers from

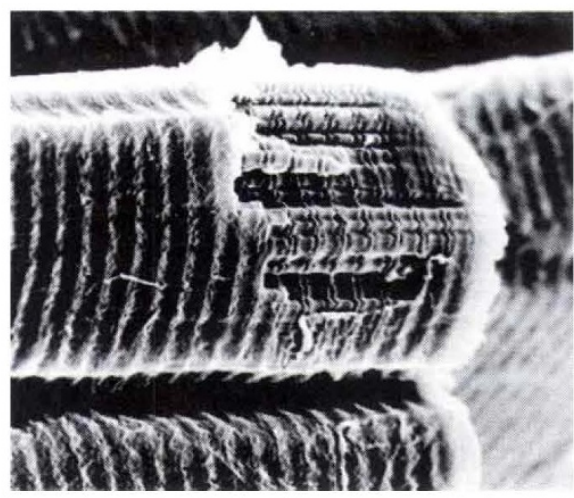

Scanning electron micrograph of human striated muscle fibres $(x \sim 1,150)$.

case reports to basic science. The reviews are excellent, particularly those related to recent advances in the cell and molecular biology of the muscular dystrophies. The journal seeks to provide a service to workers on genetic aspects by publishing a regularly revised table of gene locations and associated data for neuromuscular disorders. There are also lists of relevant, recently published papers, book reviews and conference reports.

Whether the muscle field can sustain two new journals remains to be seen. There is little direct competition: only Muscle and Nerve and perhaps Journal of Muscle Research and Cell Motility compete for a similar market. Initial submission rates look promising for Neuromuscular Disorders, which has had a rejection rate of about 50 per cent over its first two years. A more serious threat to the survival of both of these new publications will be the increasing financial constraints on institutional subscriptions. I hope the journals succeed.

Malcolm J. Jackson is in the Muscle Research Centre, Department of Medicine, University of Liverpool, PO Box 147, Liverpool L69 3BX. UK.

\section{Bridging gaps}

Jean Merrill

Advances in Neuroimmunology. Editors G. B. Stefano and E. M. Smith. Pergamon. 4/yr. £189, $\$ 290$ (institutional). £76, $\$ 117$ (personal).

ADVANCES IN NEUROIMMUNOLOGY was launched and is still manned by several pioneers in this fast-evolving interdisciplinary field. Its goal is to publish short, up-to-date reviews on the two-way communication between the nervous and immune systems, in particular its relation to evolution, development, homeostasis and pathology. The journal's competitors are few, if any; no other journal exclusively promotes reviews in this field and only one or two journals are dedicated to publishing original scientific articles on the biological links between the two systems. The recent trend among some neuroscience journals to feature special issues that specifically address neuroimmune interactions may change this unique standing of Advances. But there is clearly a need for such a review journal. Although $A d$ vances in Neuroimmunology has some growing to do, it shows great promise.

The nature of the articles has varied over the years, with some issues stressing neuroimmune themes that might otherwise have been buried in speciality journals. An example of this is the interesting issue on the evolution of neuroimmune interactions. The review articles are important not only for bringing the reader up to date; many also have an exploratory and speculative component that serves a dual purpose of bridging the gap between studies in neuroscience and immunology and fostering creative new ideas that can be addressed in the laboratory. Recent issues on highly focused areas (neuroAIDS, cytokines) have been guest-edited, breathing freshness into the contents.

Since its inception, the journal has changed shape, becoming smaller in size (trimmer in width and down to only five articles per issue). It is printed on glossy high-quality paper. Photographic illustrations, however, are few in number and mostly black and white. The trade-off is that there are no page charges to authors, and articles are published fairly rapidly. The subscription rate is a bit pricey for only four issues and prompts one to wish for more in terms of both quality and quantity. Although the luxury of subscribing to this journal may be unattainable for the individual, neuroscience and immunology departmental libraries should have copies on their shelves.

Jean Merrill is in the Department of Neurology, School of Medicine, University of California, LosAngeles, California 90024, USA. 